\author{
Journal of Al- Azhar University Engineering Sector
}

Vol. 14, No. 53, October, 2019, 1689-1695

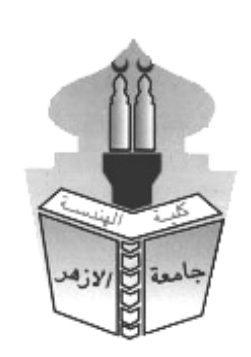

\title{
MECHANISMS OF ACHIEVING ENERGY EFFICIENCY CONSUMPTION IN EGYPTIAN SOCCER STADIUMS.
}

\author{
*Kareem Mahrous Ali ${ }^{1}$ and Nesreen Fathy Abdsallam ${ }^{2}$ \\ 1 Modern Academy For Engineering\& Technology, Cairo, Egypt \\ ${ }^{2}$ Faculty of Engineering Mattaria, Helwan University, Cairo, Egypt \\ *Corresponding author E-mail: Architectkareemmahrous87@gmail.com
}

\begin{abstract}
ABASTRACT
This research discusses how to achieve Energy efficiency consumption, which is considered as one of the most important contemporary issues globally and locally,because of the increase in the prices of energy \& the limited resources and carbon emissions which are harmful to the environment.Sustainable stadiums are considered as a requirements of the world's advocated by the International Federation of Football (FIFA) Serving community issues and solving problems, despite this importance, the local stadiums are traditional and unsustainable, especially with regard to achieve Energy efficiency consumption, This is contrary to Egypt desire to achieve overall sustainability by 2030 . This research discuss the mechanisms of energy efficiency consumption in Sustainable Football Stadiums in order to benefit from raising the efficiency of the local stadiums and developing them to become sustainable in terms of Energy efficiency consumption.
\end{abstract}

KEY WORDS: Sustainable Stadiums,Football,Energy,Efficiency,Consumption Negative Design,Positive,Design,Renewable,Energy,hotovoltaic,Cells.Wind

Turbine

$$
\begin{aligned}
& \text { آليات ترشيد استهلاك الطاقة فى استادات كرة القدم المصرية }
\end{aligned}
$$

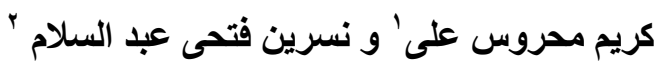

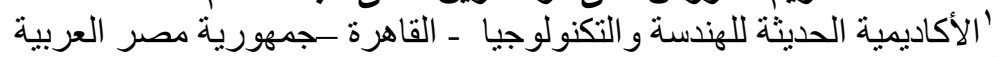

$$
\begin{aligned}
& \text { †هندسة المطرية-جامعة حلوان- حلوان ـ القاهرة - جمهورية مصر العربة العربية }
\end{aligned}
$$

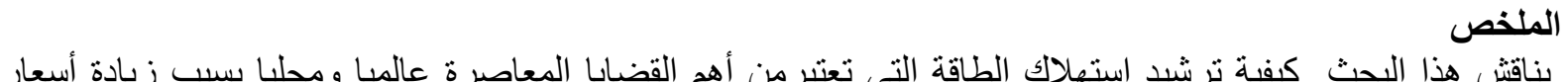

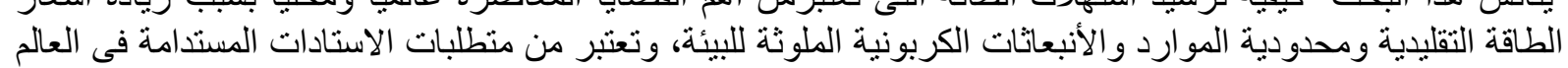

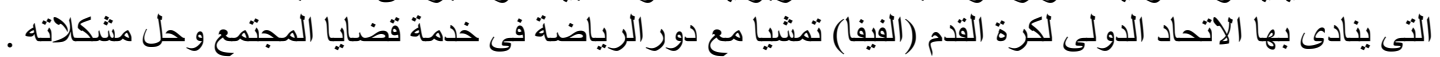

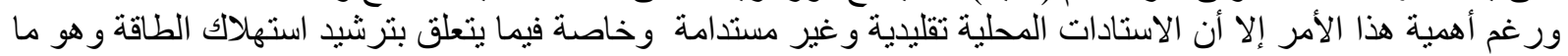

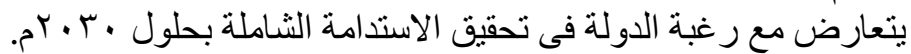

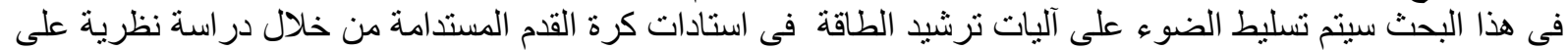

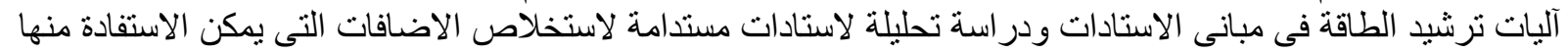

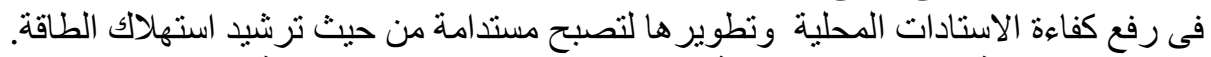

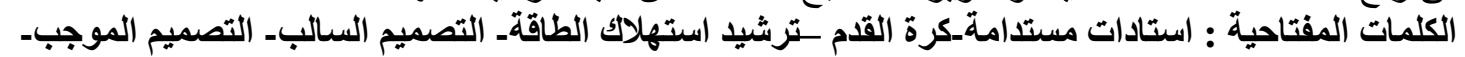

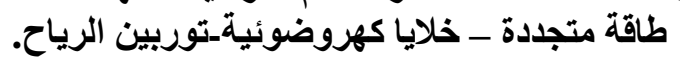


يعتبر ترشيد استهلاك الطاقة أحد المنطلبات الأساسية فى بناء الاستادات الجديدة لأهمية ذلك في خفض تكاليف التشغيل و الحد من الطلب على الطاقة التقليدية التى تتسم بمحدودية الموارد وارتفاع تكلفة شر اءها وتلوثة التهات البيئة نتيجة الانبعاثات الكربونية الناتجة عن انتاجها واستخدامها. ونتيجة لأهمية هذا الأمر أصبح من منطلبات تحقيق استدامة المبانى عامة والاستادات خاصة قدرتها على ترشيد الطاقة و التى تعتبر أكثر عناصر التقييم أهمية فى معظم نظم الاستدامة العالمية مثل (LEED,BREEAM) و الثانى نرتبيا بعد الأني

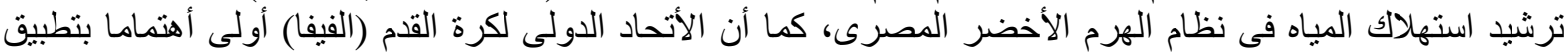

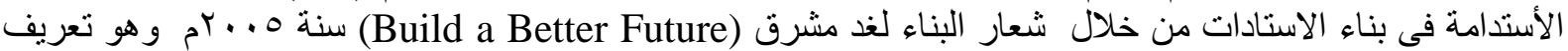

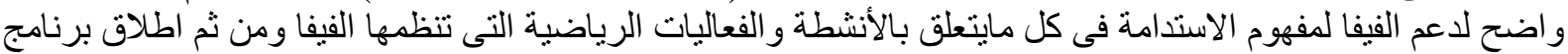

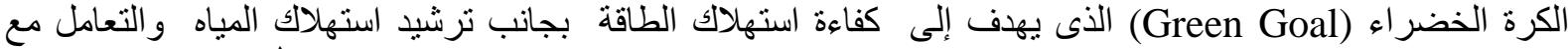

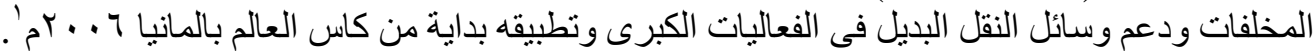

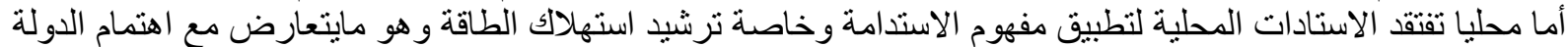

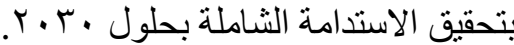

المشكلة البحثية

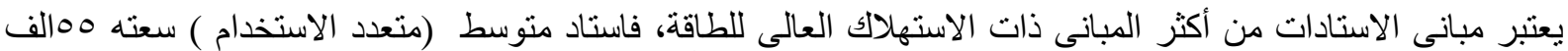
مقعد يستهلك فعليا أكثر من ·.الاف ميجاوات من الطاقة سنويا؟، مثال آخر وهو استاد رئاد رويال بافوكينج ( Royal)

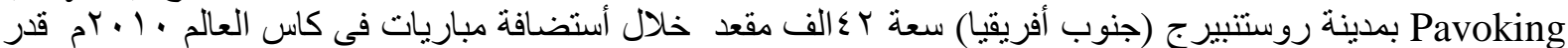

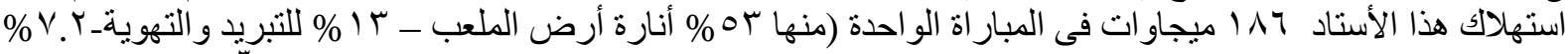

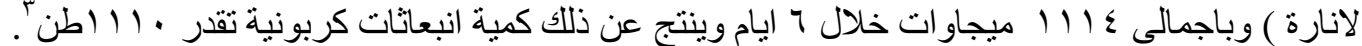

فرضية البحث ترشيد استهلاك الطاقة والاعتماد على طاقة متجددة بساهم فى خفض تكاليف التشغيل للاستاد والحد من الانبعاثات الكربونية الضارة بالبيئة. هدف البحث م الاستهلاك الرشيد للطاقة فى استادات كرة القدم. • • نقل خبرة التجارب العالمية مع الاستادات المستدامة إلى الاستادات المحلية فيما يتعلق بترشيد استهلاك الطاقة. منهجية البحث

تنقسم الدر اسة إلى ثناثة أجز اء رئيسية هما الدر اسة النظرية و الدر اسة التحليلية و الدر اسة التطبيقية

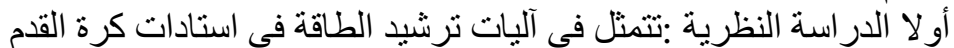

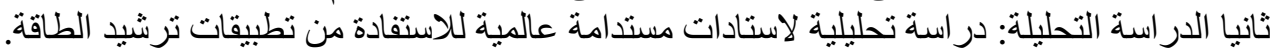

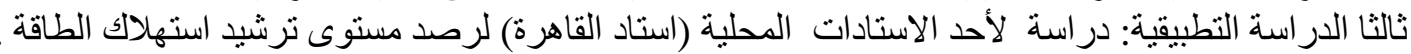

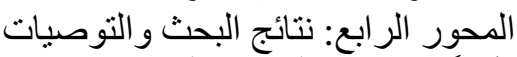

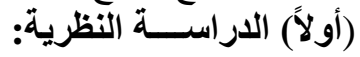

أـآليات ترشيد الطاقة فى استادات كرة القدم

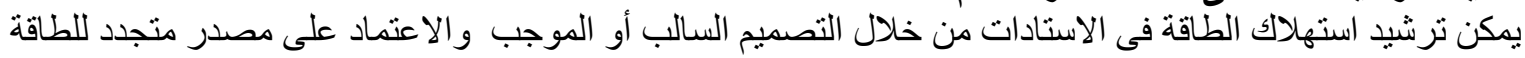

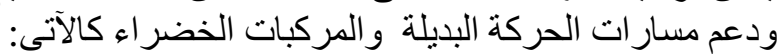
• التصميم السلبى: توظيف استراتيجات التصميم السلبى القائم على التصميم المعمارى المدروس بيئيا دون الاعتماد على التى النظم الميكانيكية و التكنولوجية تونفية المساعدة مثل:

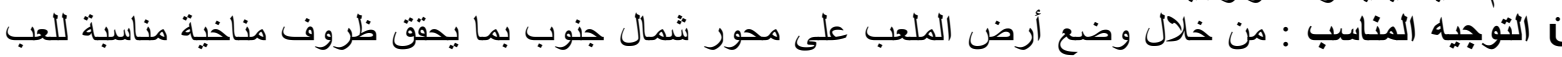

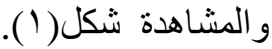

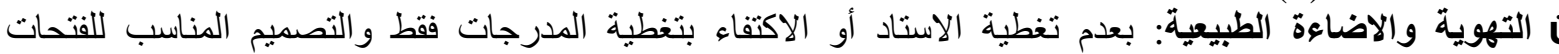

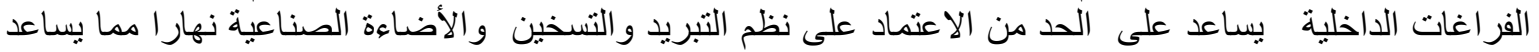
على ترشيد استهلاك الطاقة

${ }^{1}$ FIFA,2011," Football Stadiums, Technical recommenda ons and requirements", $5^{\text {th }}$ edition.

2 Thomas Smulders," Green stadium as green as grass ",M aster Thesis, Utrecht University,2012

${ }^{3}$ South Africa 2010,Energy",Report,2011 
ـ تنسيق الموقع العام: من خلال زر اعة أسطح المبانى الملحقة بالاستاد أو سقف الاستاد وزيادة نسبة العناصر النباتية

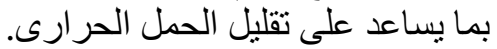
ر معالجة الواجهات المعرضة للشمس: باستخدام الكواسر أو تصميم الفتحات أو استخدام الالوان الفاتحة أو..الخ بما

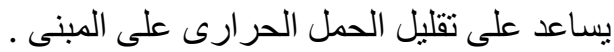

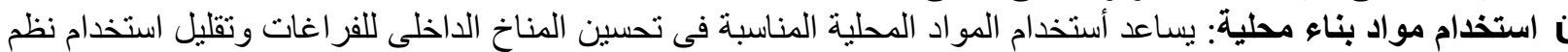

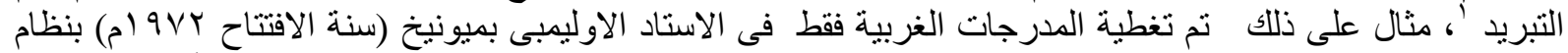

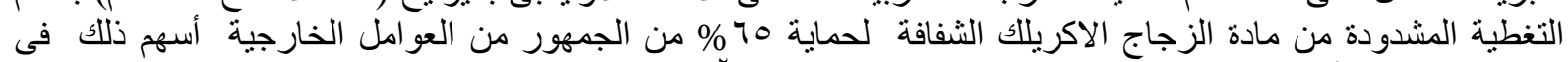

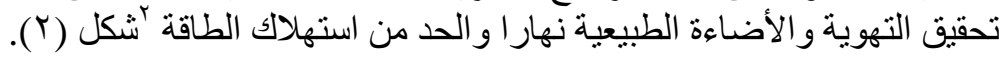
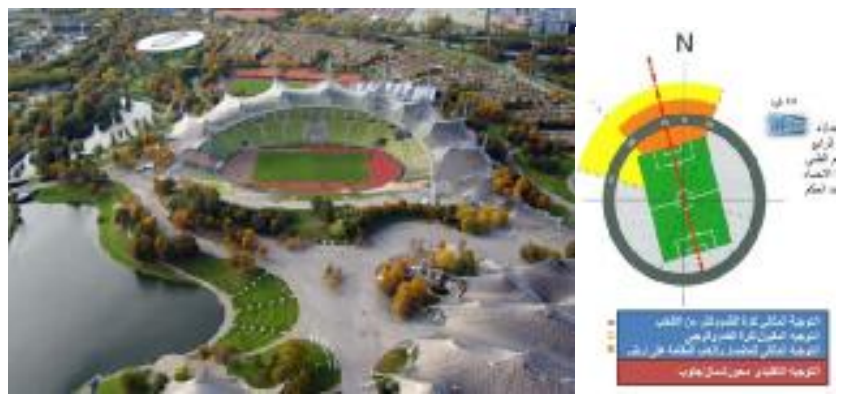

شكل (1) أسكتش يوضح التوجيه المناسب شكل(r) الأستاد الأوليمبى بموينخ نموذج للتصميم السالب المبال المصدر: Www.maber.co.uk

التصميم الأيجابى : نوظيف أستر اتيجات التصميم الإيجابى القائم على الاعنماد على النظم أو التركيبات الذكية المتطورة

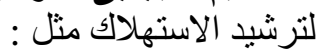

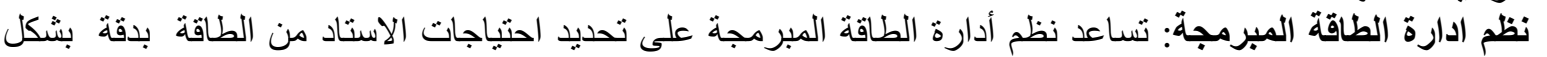

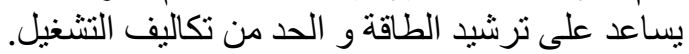

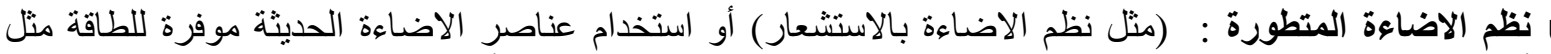

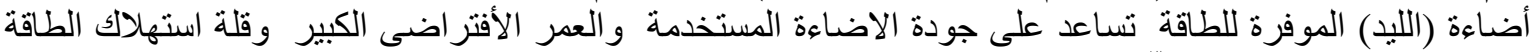

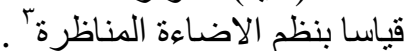
د. استخدام نظام لإستغلال الحرارة المفقودة كمصدر للطاقة بساعد على تقليل احتباج الأستاد من الطاقة.

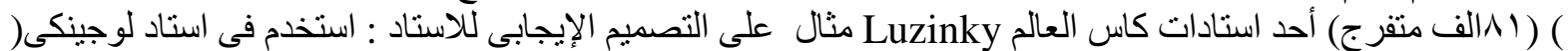

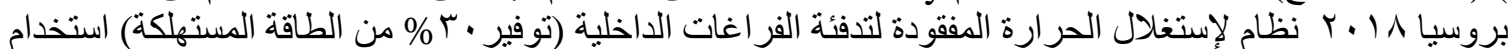

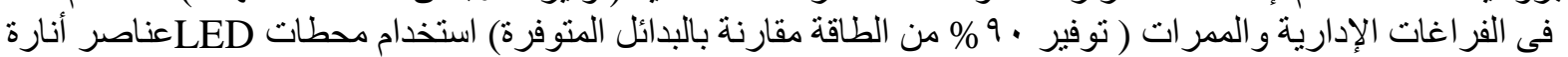

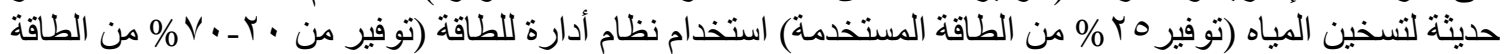
المستخدمة).

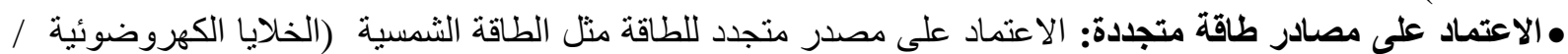

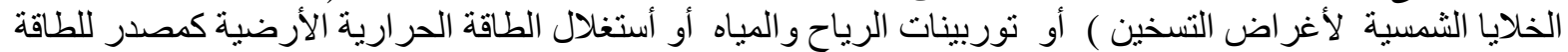

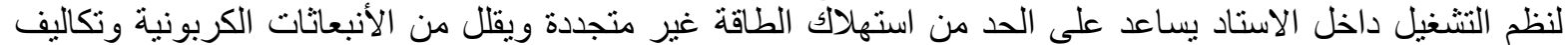
التشغيل. مثنال على ذلك استاد عالم الألعاب (World Games Stadium) فى تايوان (00 الف مقعد) يمثل نموذجا للاستادات

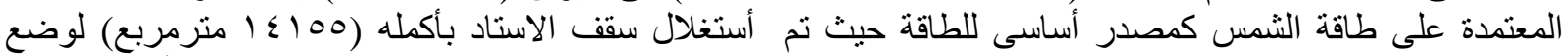

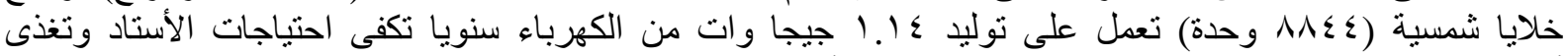

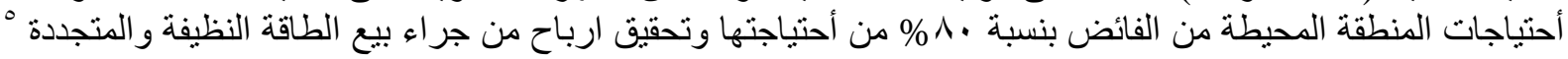

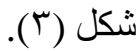

\footnotetext{
${ }^{1}$ Sam Kubba,2012,"Green Building Design and Construc on",Elsevier.

2 www.archdaily.com

${ }^{3}$ Sam Kubba,2012,"Green Building Design and Construc on",Elsevier

${ }^{4}$ FIFA,"M ore Sustainable Stadium" 2 nd technical report the implementation of environmental, energy- and resource-effi cient design solu ons for the stadiums of the 2018 FIFA World Cup Russia,2016.

${ }^{5} \mathrm{~h}$ ps://www.archdaily.com/22520/taiwan-solar-powered-stadium-toyo-ito.
} 

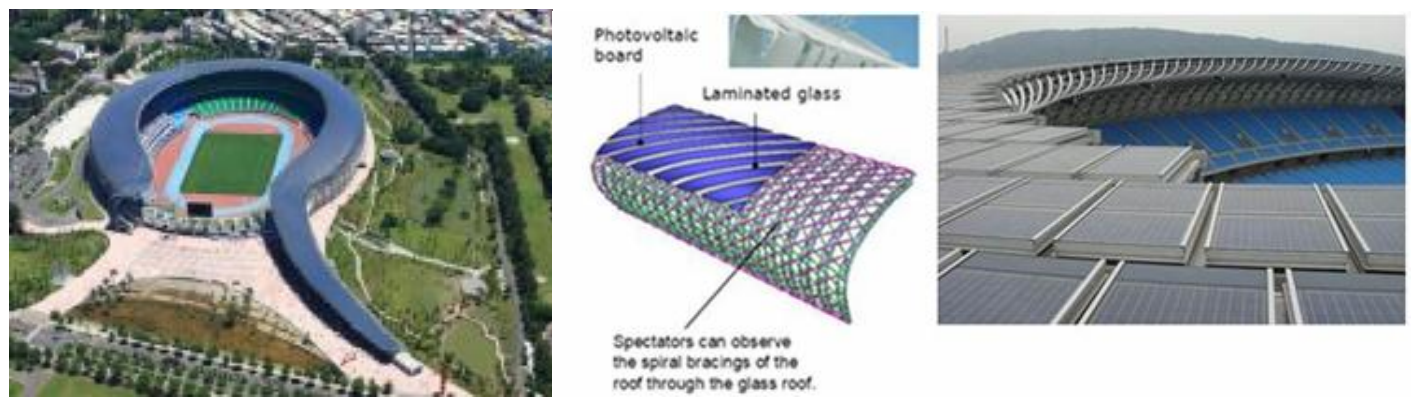

شكل (r):صورة لاستاد عالم الألعاب تغطية السقف كاملا بالخلايا الكهروضوئية WWw.archdaily.com المصدر

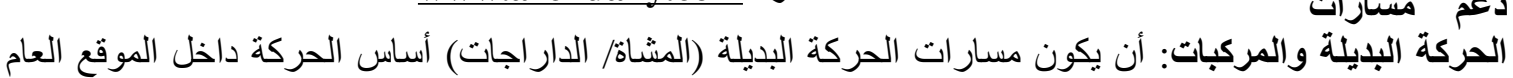

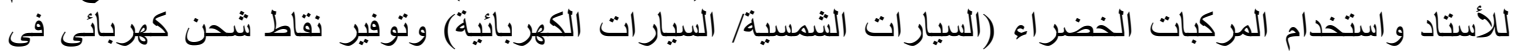

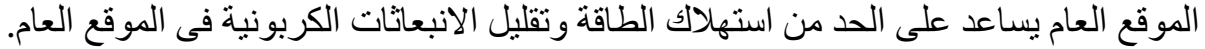

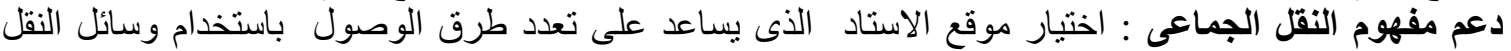

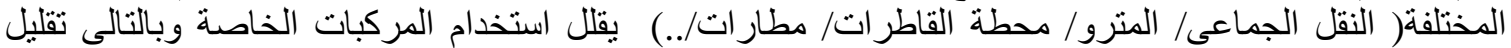

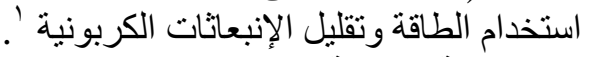

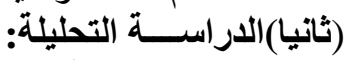
ب- استادات مستدامة موفرة للطاقة. سيتم تحليل أربعة من الاستادات المستدامة تم اختيارهات التميز ها في تحقيق آليات مستدامة لتوفير الطاقة، كما بجدول (1) و التى يمكن الأستفادة منها فى تطوير وتحسين الاستادات المحلية لكى تكون موفرة للطاقة.

جدول (1) الاستادات المستدامة المختارة المصدر: www.archdaily.com بتجميع الباحث

\begin{tabular}{|c|c|c|c|c|}
\hline التقييم & التشغيل & السعة & البلد البل & أسم الأستاد \\
\hline من أوائل الاستادات المستات عالميا & 1997 & ؛ هالف مقعد & امسترد/م_هولتدا & $\begin{array}{c}\text { امستردام أرينا } \\
\text { Amsterdam Arena }\end{array}$ \\
\hline $\begin{array}{c}\text { LEED (New } \\
\text { construction) Gold }\end{array}$ & $r \ldots 1$ & ا9 الف مقعد & بكين- الصين & $\begin{array}{c}\text { عش الطائر } \\
\text { Bird Nest }\end{array}$ \\
\hline جوائز متعددة & $r \ldots q$ & ه ^الف مقعد & دربان-ج أفريقيا & $\begin{array}{c}\text { موسى مبيدا } \\
\text { Mouse Membeda }\end{array}$ \\
\hline BS8901-2009 & $r+1$. & • هالف مقعد & دبلن-ايرلتدا & $\begin{array}{c}\text { افيفا } \\
\text { Aviva }\end{array}$ \\
\hline
\end{tabular}

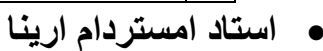

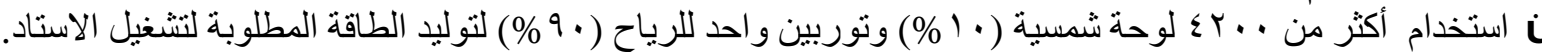

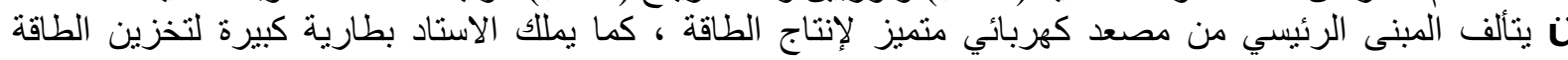

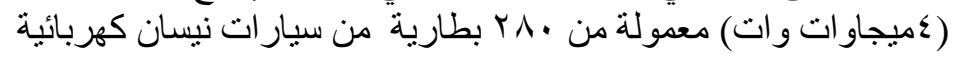
ر توفير شحن للسيار ات الكهربائية فى الموقع العام للاستاد.

ر يتم استخدام هالك الحر ارة المتبقية للحفاظ على الملعب من حالات الصقيع

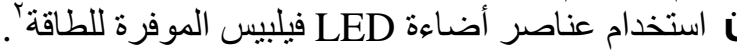

${ }^{1}$ UEFA ,"GUIDE TO QUALITY STADIUM S,2011,p36-39

${ }^{2} \mathrm{~h}$ ps://www.essma.eu/news/item/857-innovation-sustainability-and-quality-as-strategic-pillars-ofamsterdam-arena 


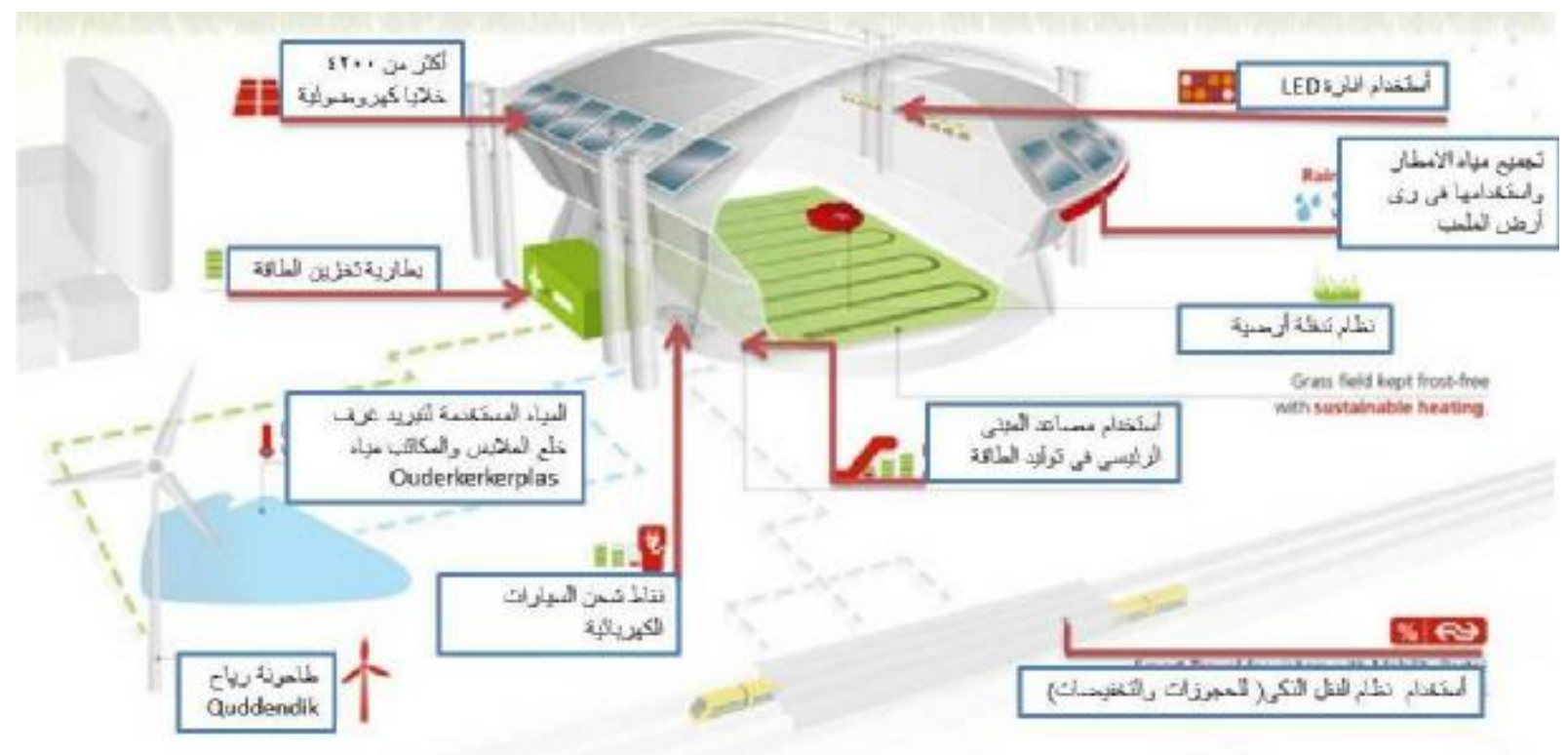

شكل ؛ التطبيقات المختلفة لتوفير الطاقة فى استاد امستردام ارينا المصدر: https://www.essma.eu ر تصميم مبنى الاستاد بما يساعد على الاضاءة والتهائة التهوية الطبيعية بالتالى الحد من استخدام النظم الميكانيكية والاضاءة

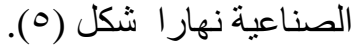
ر استخدام تكنولوجيا منطورة لإستخدام الطاقة الحرارية الأرضية كمصدر للطاقة المتجددة وضبط درجة حرارة الاستاد

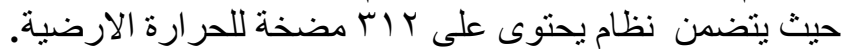

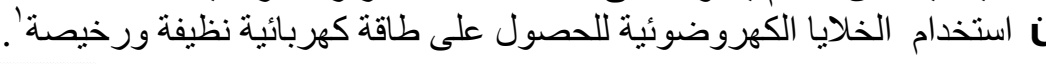
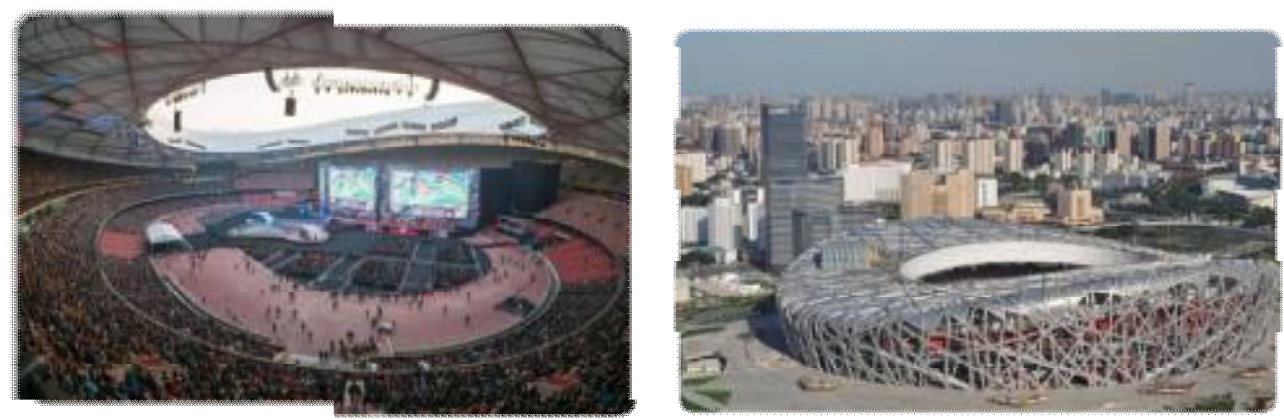

شكل (•) استاد عش الطائر والاعتماد على التهوية والاضاءة الطبيعية المصدر:

• استاد موسى مبيدا:

الاعتماد على الاضاءة و التهوية الطبيعية فى تصميم الاستاد شكل (T).

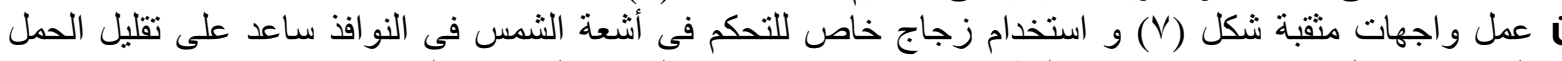

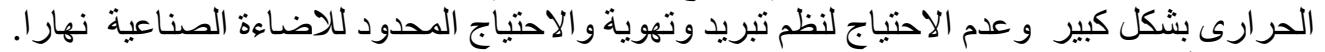

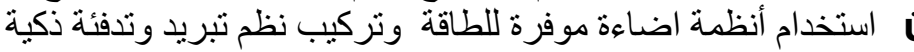

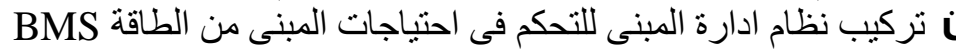

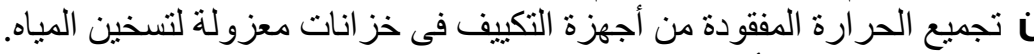

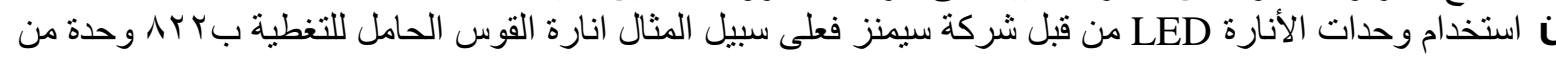
توفر • rLED Beam Spotlight

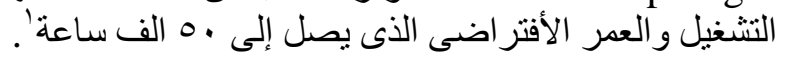

${ }^{1}$ h ps://www.renewableenergyworld.com/arc les/2008/08/beijing-olympics-show-chinasrenewable-energy-aspirations-53240.html 

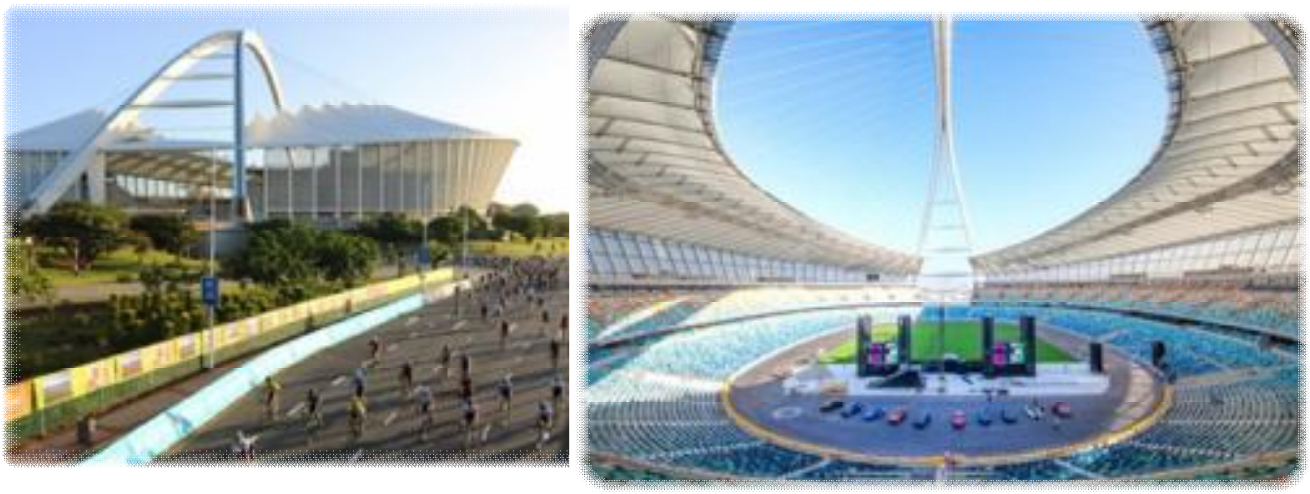

شكل( آ) التهوية والاضاعة الطبيعة للمدرجات والملعب شكل(V) الواجهات المثقبة الثفافة للاستاد المصدر: المصدر: المبكان

• استاد افيفا

ه تغطية المدرجات فقط شكل(^) و الو اجهات بالو اح الكاربونيت الثفافة تساعد على الاضناءة و التهوية الطبيعية شكل (9). استخدام نظام ذكى للاضـاءة ونظام تحكم فى التبريد والتسخين ساهم فى انخفاض استهالاك الكهرباء بعد سنة من تشغيل الاستاد بنحو

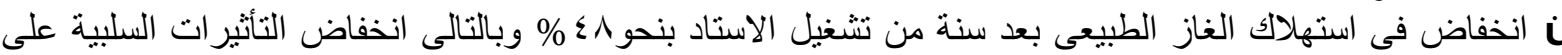

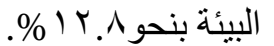

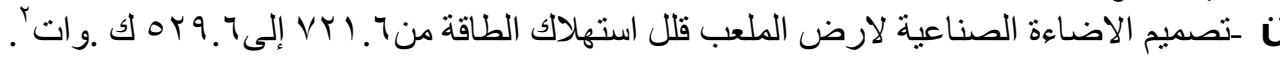

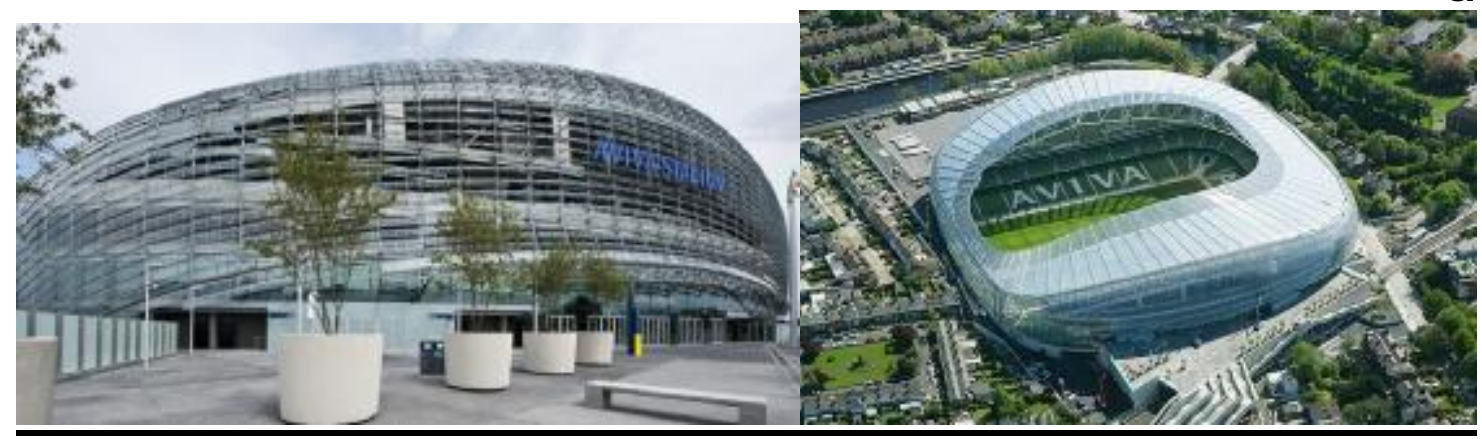

شكل (^) تغطية المدرجات فقط بالواح الكاربونيت الشفافة شكل(9) استخدام الواح الكاربونيت فى تغطية الواجهات المصدر:Www.archdaily.com المصدر:

يمكن تلخيص التطبيقات الخاصة بترشيد استهلاك الطاقة فى الاستادات المختارة

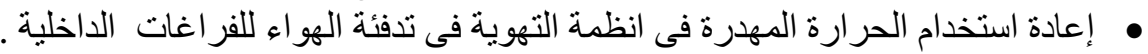

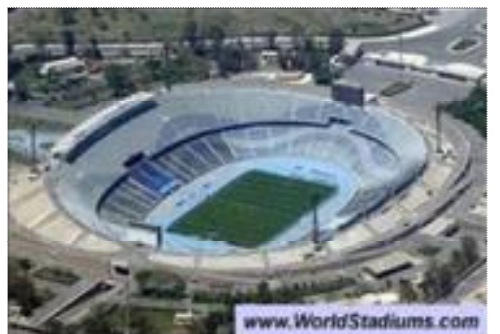

شكل( • 1 ) أستاد القاهرة الدولى المصدر:WWW.Worldstadiums.com

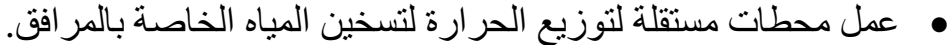
• • استخدام نظم تحكم و ادارة الطاقة المستهلكة. • الجمع بين مصدر متجدد للطاقة أو أكثر لإمداد الاستاد من الطاقة الطاقة.

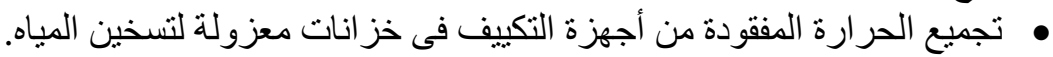
• تصميم الاضاءة الصناعية لارض الملعب تقلل استهلاك الطاقة.

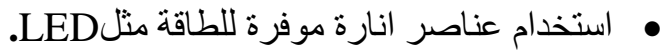
ثالثا :الدر استة التطبيقية

ج-رصد مستوى ترشيد أستهلاك الطاقة في الأستادات المحلية.

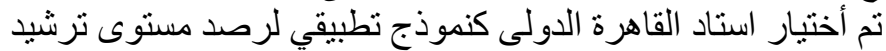
أستهلاك الطاقة من حيث نقاط القوة و الضعف الضك

\footnotetext{
${ }^{1}$ https://www.siemens.com/press/pool/de/events/2011/corporate/2011-11-african/factsheet-mosesmabhida-station-stadium-e.pdf.

${ }^{2}$ https://www.mse.ie/portfolio-items/aviva-stadium-sportslighting-dublin.
} 


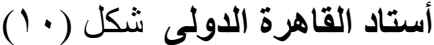

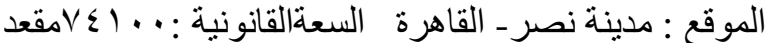

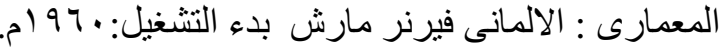

نتائج الزيارة الميدانية

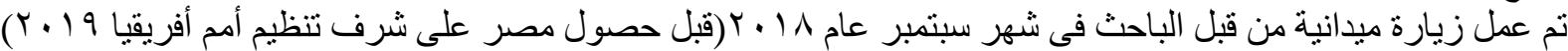

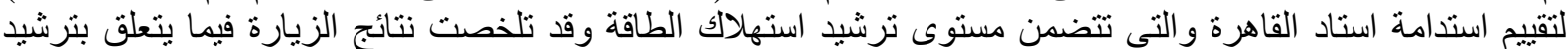

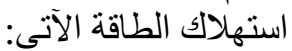

\section{• نقاط الضعف الخاصة بترشيد الطاقة:}

ر خمس الطاقة المستخدمة الديزل واربع أخماس من كهرباء المنطقة (طاقة غير متجددة) لتوفير احتياجات الاستاد من

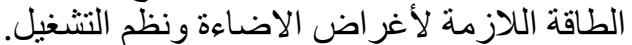

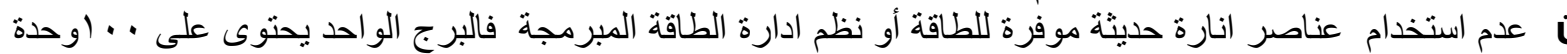

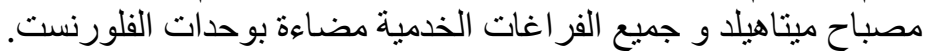

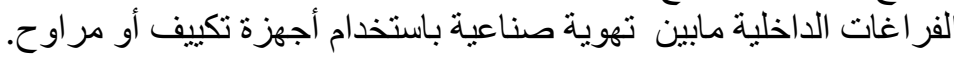

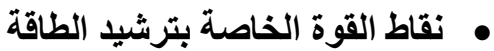

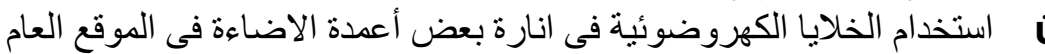

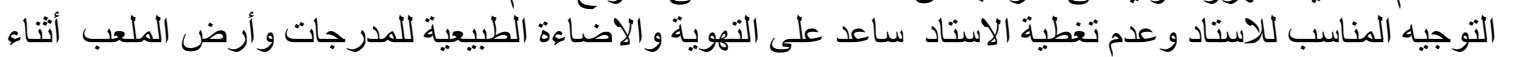

الو اجهات المفر غة ساعدت على الاضلاءة و التهوية الطبيعية للممر ات و المداخل شكل (1) (1).

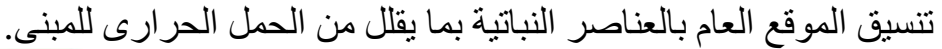
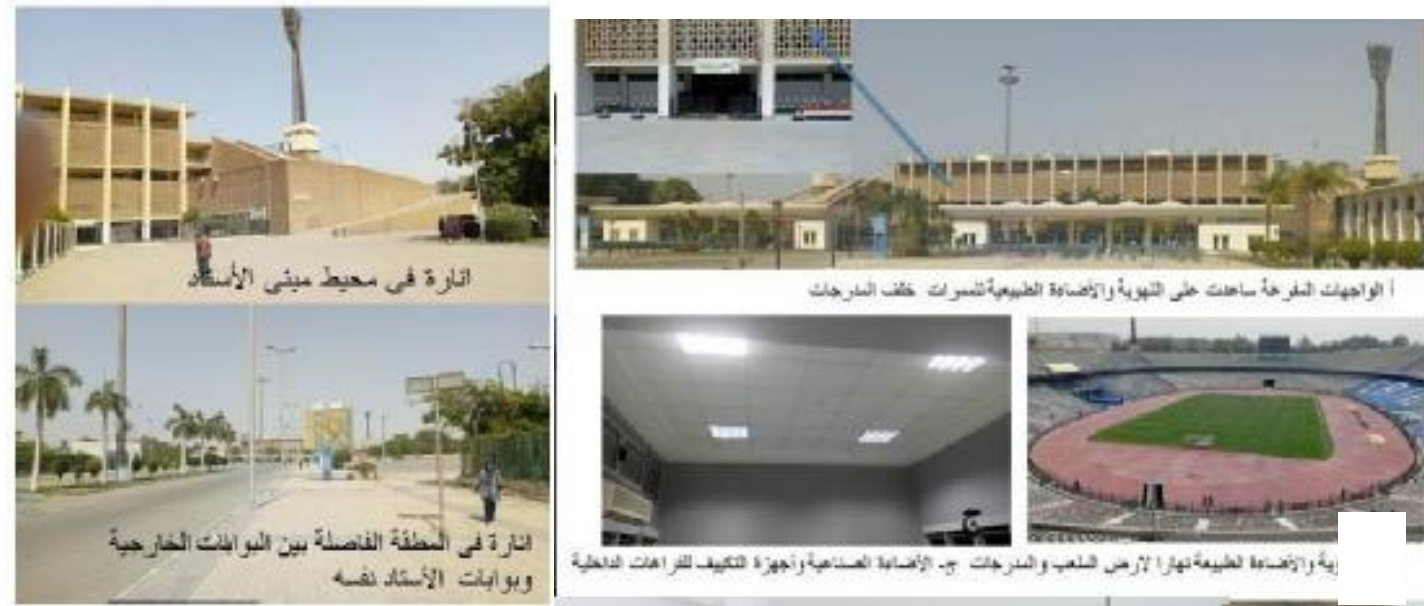

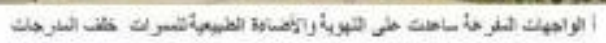

شكل(1) 11 ) صور ترصد بعض نقاط القوة والضعف الخاصة بترشيد الطاقة المصدر:الباحث

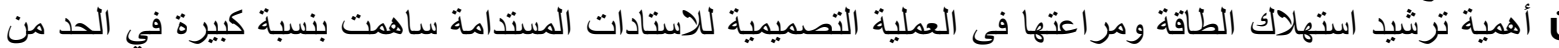

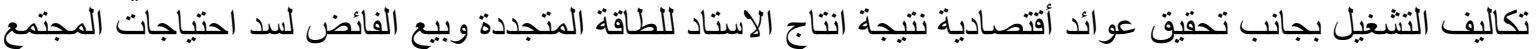
المحيط مثل استاد عالم الألعاب بتايوان.

ـ استاد القاهرة الدولى هو استاد تقليدى من حيث ترشيد استهلاك الطاقة و أكتفى ببعض تطبيقات التصميم السالب (التهوية

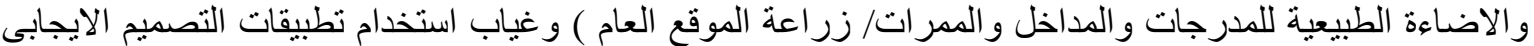

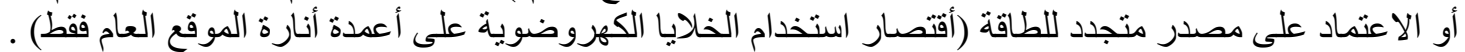

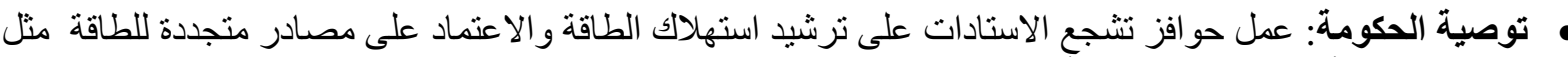

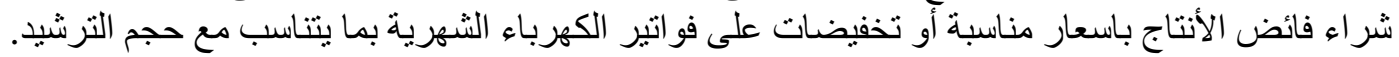

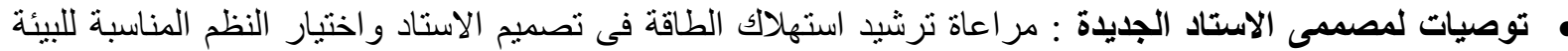

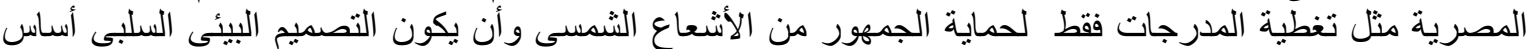

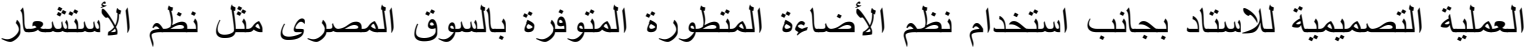




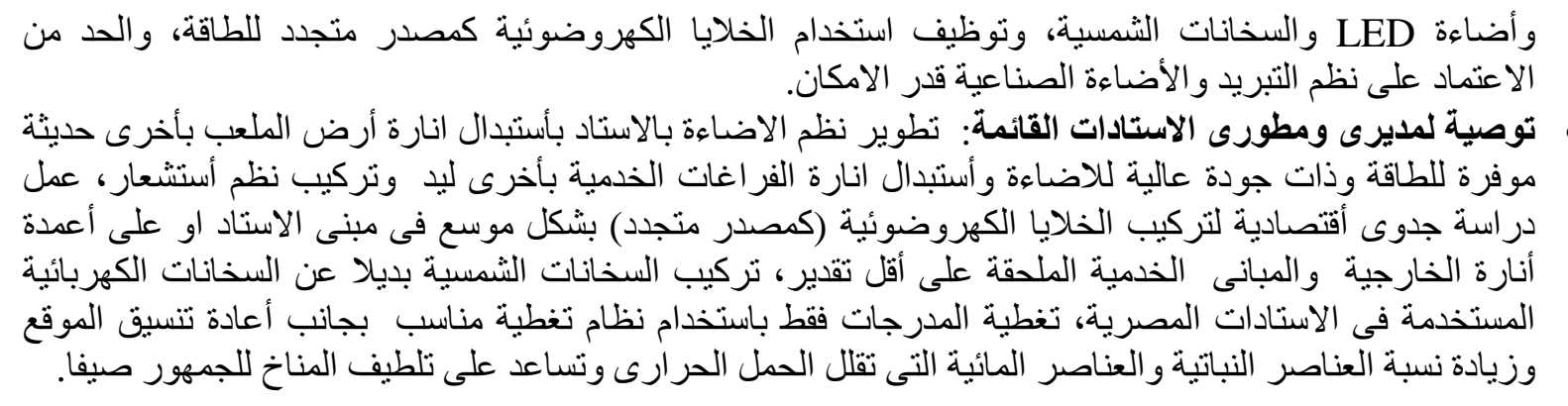

1. Thomas Smulders,2012" Green Stadium As Green As Grass ",Master Thesis, Utrecht University,P33

2. UEFA 2011,,Guide To Quality Stadium.

3. FIFA,2011," Football Stadiums, Technical Recommendations And Requirements", $5^{\text {th }}$ Edition.

4. FIFA,2011South Africa 2010,Energy.

5. FIFA,2016,"More Sustainable Stadium"2 ${ }^{\text {nd }}$ Technical Report The Implementation Of Environmental, Energy- And Resource-Effi Cient Design Solutions For The Stadiums Of The 2018 FIFA World Cup Russia.

6. Https://Www.Archdaily.Com/22520/Taiwan-Solar-Powered-Stadium-Toyo-Ito.Https://Www.Essma.Eu/News/Item/857-Innovation-Sustainability-And-Quality-As-StrategicPillars-Of-Amsterdam-Arena.

7. Https://Www.Mse.Ie/Portfolio-Items/Aviva-Stadium-Sportslighting-Dublin-4.

8. Https://Www.Renewableenergyworld.Com/Articles/2008/08/Beijing-Olympics-ShowChinas-Renewable-Energy-Aspirations-53240.Html.

9. Https://Www.Siemens.Com/Press/Pool/De/Events/2011/Corporate/2011-11African/Factsheet-Moses-Mabhida-.

10.Sam Kubba,2012,"Green Building Design And Construction", Amsterdam, Holland, Elsevier. 